\section{Characteristics of progression of early Age-related macular degeneration: the Cardiovascular Health and Age-related maculopathy Study}

G Tikellis', LD Robman ${ }^{1,2}$, P Dimitrov1 , C Nicolas¹, CA McCarty ${ }^{3,1}$ and RH Guymer ${ }^{1,2}$

\begin{abstract}
Aims To determine the risk of age-related macular degeneration (AMD) progression posed by the presence of each early AMD characteristic.
\end{abstract}

Methods A prospective cohort study of 254 participants aged 50 years and older, all with early AMD features at their baseline visit followed for an average of 7 years.

Stereoscopic colour fundus photographs were graded for early AMD features using the International Classification System. AMD status was stratified into six exclusive levels along a continuum of disease severity according to drusen type, pigmentary abnormalities, or late AMD. Progression was assessed according to three definitions: a change between or within a severity level, or by side by side grading.

Results The progression rate of early AMD ranged between 3.4 and $4.67 \%$ per annum depending upon the definition used. In total, $15(6 \%)$ cases progressed from early AMD to the late complication of AMD. After controlling for age and smoking, cases with soft indistinct drusen at baseline were at a greater risk of progressing from early to late AMD than were cases without this characteristic $(\mathrm{OR}=3.72,95 \% \mathrm{CI} 1.20-11.54$; $P=0.02$ ).

Conclusion Our proposed definitions of AMD progression give rates that are consistent with current knowledge of progression and its determinants. Each early AMD characteristic conveys its own risk of progression to an eye, with soft indistinct drusen carrying the greater risk. An international consensus on what defines AMD progression would greatly help the research community when trying to assess the importance of new risk factors and the effectiveness of novel interventions.

Eye (2007) 21, 169-176. doi:10.1038/sj.eye.6702151; published online 26 May 2006

Keywords: age-related macular degeneration; age-related maculopathy; progression; drusen

\section{Introduction}

Age-related macular degeneration (AMD) remains the most common cause of irreversible vision loss in people aged 50 years and older in communities of the industrialised world. If our ability to treat or prevent this disease does not improve, there will be a doubling in the number of people with AMD by the year $2020 .^{1}$

A major limitation in the current knowledge of the disease is the lack of understanding of the aetiology and natural progression of AMD in elderly populations. Numerous populationbased epidemiologic studies have examined the incidence and progression rates of AMD. ${ }^{2-7}$ In these studies, the focus has primarily been on the progression of AMD from the early to the late stages of the disease. A better understanding of what defines progression of early AMD lesions would provide further information regarding the natural development of the disease. A universally agreed upon definition of progression stages, along the lines of the International classification of fundus features of AMD, would allow researchers to better define stages of the disease, monitor
${ }^{1}$ Department of Ophthalmology, Centre for Eye Research Australia, University of Melbourne, East Melbourne, Victoria, Australia

${ }^{2}$ Department of Epidemiology and Preventive Medicine, Monash University, Melbourne, Australia

${ }^{3}$ Marshfield Clinic Research Foundation, Wisconsin, USA

Correspondence: G Tikellis, Department of Ophthalmology,

Centre for Eye Research Australia, University of Melbourne, Locked Bag 8, East Melbourne,

Victoria, 8002, Australia Tel: + 61399298571 ; Fax: +61 396623859 . E-mail: gtike@ unimelb.edu.au

Received: 1 June 2005 Accepted: 17 September 2005

Published online: 26 May 2006 
natural progression towards end-stage disease, and give a firm foundation upon which to base strategies for intervention at particular stages and to document efficacy of these interventions.

Unfortunately at present there is no universally agreed upon classification of orderly progression in early AMD. Thus in this paper, we describe how we scored all characteristics of the maculae and derived step-wise increases in severity based upon the knowledge and understanding we currently have about progression and devised three definitions of progression.

There have been three major studies that have used various classification systems to assess and define AMD progression. The Beaver Dam Eye Study was one of the first studies to report on the progression of age-related maculopathy. ${ }^{4}$ Using the Wisconsin Age-related Maculopathy Grading System to assess AMD status, progression was defined for each lesion based on the presence of the lesion at baseline and its appearance in two or more additional subfields at follow-up. In this study, the appearance of a new lesion was categorised as an incident case rather than a case of increased AMD severity and thus progression.

In the Rotterdam Study, Klaver et $a l^{2}$ used the International Classification System for AMD and stratified the disease into four exclusive stages according to drusen type and the presence of pigmentary abnormalities in the worse eye. In this study, progression was defined as a worsening to the next or a higher stage of AMD. Although intermediate drusen (soft drusen of size $63-125 \mu \mathrm{m}$ ) are described in the International Grading System as a transitional step between hard and soft drusen, the Rotterdam Study did not allocate this drusen type in a separate category and did not mention this type in any of their AMD stages. Thus, it is not clear whether any drusen larger than 63 but smaller than $125 \mu$ m were incorporated into the analyses.

In the Age-related Eye Disease Study (AREDS), ${ }^{6}$ cases were classified into four categories based on drusen type and number, but unlike any previous study a bestcorrected visual acuity score was included into the characteristics that determined stage. The main outcome measures of the study were the photographic assessment of progression to or treatment for advanced AMD and at least a moderate visual acuity loss from baseline.

Unlike the well-categorised grading systems for documenting AMD features, there is no internationally recognised system for determining what changes in fundus characteristics represent an orderly progression of the disease towards the end stage.

Until we have a better understanding of what constitutes an orderly progression of the disease and which lesions are associated with greatest risk for developing late AMD, we will be greatly disadvantaged when planning intervention studies and trying to measure the impact of new treatment strategies on progression.

The purpose of this paper was to attempt to define progression incorporating past attempts along with our current understanding of which characteristics constitute risk of end-stage disease. Our purpose was then to examine the progression rate of AMD in our study of cardiovascular risk factors in age-related maculopthy to determine which lesions present in early AMD appear to contribute significantly to the risk of progression.

\section{Materials and methods}

\section{Participants}

The Cardiovascular Health and Age-related Maculopathy Study (CHARM) was designed to examine potential risk factors, including newly identified risk factors for cardiovascular disease, that might be associated with AMD and its progression. ${ }^{8}$ The study protocol was approved by the Royal Victorian Eye and Ear Hospital Human Research Ethics Committee (project \# 99/372H). Written informed consent was obtained from each study participant.

The progression study enrolled participants with early AMD features from two previous studies conducted in Melbourne: (1) the Melbourne Visual Impairment Project (VIP), a population-based study of 3271 men and women aged 40 years and older conducted from 1992-1994 to examine the causes of vision loss in Australia. ${ }^{9}$ The Vitamin E, Cataract and Age-related Maculopathy Study (VECAT), a prospective, randomised controlled study of 1193 volunteers aged 55 years and older, established to determine the efficacy of vitamin E supplementation on the incidence and progression of cataract and AMD. ${ }^{10}$ The VECAT Study was conducted from 1994 to 2000 and found that vitamin $E$ had no effect on the course of AMD or cataract ${ }^{11,12}$ and therefore participants identified as having early AMD features from this study were deemed eligible to participate in the CHARM Study. Both studies were conducted at approximately the same time in Melbourne, and employed similar study methodology and protocol to document retinal features and risk factors associated with AMD.

It was from these two large cohorts that subjects with early AMD were invited to participate in the CHARM Study. ${ }^{8}$ Such cohorts provided an opportunity to follow early AMD cases over an average period of 7 years where they were re-examined as part of the CHARM Study. Methods used to identify and describe the cohort have been described previously ${ }^{8}$ but will be outlined briefly below. 


\section{Procedures}

Participants deemed eligible to participate in the CHARM Study were examined during 2001-2002. The visit included a clinical examination, macular photography, the administration of questionnaires, and the taking of a fasting blood sample.

The clinical tests involved an ophthalmic examination as well as vascular and selected anthropometric measurements. Best-corrected visual acuity, visual function testing and cognitive function testing formed part of the ophthalmic examination. Stereo photography of the macula was performed following pupil dilation using methods similar to that used in both the VIP and VECAT studies. ${ }^{9,10}$ Cardiovascular assessment allowed measurement of new cardiovascular risk factors such as systemic arterial compliance, pulse wave velocity, and intima media thickness. Anthropological measurements and dietary assessment of various nutrients along with a fasting blood sample provided information regarding numerous AMD and cardiovascular disease risk factors.

\section{Assessment of AMD on macula photographs}

Grading of macula photographs to assess AMD status was based on the International Classification and Grading System for AMD. ${ }^{13}$ Details of the grading procedures are presented elsewhere. ${ }^{14,15}$

In brief, following the specifications outlined by the International group, a grid consisting of three circles concentric with the centre of the macula that represent, respectively, the central, middle, and outer subfields was used to assist with the grading of early and late AMD lesions. The central, middle, and outer circles have diameters of 1000, 3000, and $6000 \mu \mathrm{m}$, respectively. This grid was developed by the Wisconsin ARM Grading System ${ }^{16}$ and has been used in other eye studies. ${ }^{17}$ Each eye was graded independently of the fellow eye. The assessment consisted of a lesion-by-lesion evaluation documenting the absence or presence of any feature, then its size, location, total number and overall area covered (with respect to each of the three circles) within the boundaries of the grid that corresponded to an area of $6000 \mu \mathrm{m}$ diameter across the macula, centred on the fovea.

AMD features graded in each eye included five different drusen types:

- hard drusen: any drusen $<63 \mu \mathrm{m}$ or drusen of size $\geq 63$ and $<125 \mu \mathrm{m}$ with distinct edges;

- soft intermediate drusen: $\geq 63$ and $<125 \mu \mathrm{m}$ with indistinct edges;

- soft distinct drusen: $\geq 125 \mu \mathrm{m}$ with distinct edges;

- soft indistinct drusen: $\geq 125 \mu \mathrm{m}$ with indistinct edges.
Retinal pigment epithelium changes (hyper- and hypopigmentation), geographic atrophy, and neovascular AMD were also noted. Circles of defined size (63, 125, $175,250 \mu \mathrm{m}$ in diameter) printed onto clear plastic were used to estimate size of drusen and areas involved by drusen, increased retinal pigment, and retinal pigment epithelial depigmentation. Total number of each drusen type was categorised as $1-9,10-19$, or 20 or more. Area covered by drusen in each of the three circles was categorised as $<10,<25,<50$, or $50 \%$ or more as outlined in the International Classification System. ${ }^{13}$

\section{Definition of disease severity}

As outlined in the introduction, our aim was to examine each early AMD characteristic to determine the risk of progression associated with its presence. As in the Rotterdam and AREDS studies, we have used a stratification system for AMD and have based our study primarily on the system outlined in the Rotterdam Study with some modifications. The first allowed the inclusion of soft intermediate drusen (a class of drusen omitted in the Rotterdam system) and the second was the segregation of hyperpigmentation from hypopigmentation of the retinal pigment epithelium (RPE). We believe it is important to differentiate specifically between hyperand hypopigmentation of the retinal pigment epithelium as they are believed to have different implications for severity and convey different risks of progression. By incorporating these modifications, we have expanded our levels to accommodate all possible stages of the disease, allowing us to examine each characteristic's contribution to the progression of AMD.

Thus, AMD status at baseline and at follow-up was stratified into six exclusive stages of disease. For each eye, AMD was defined as follows:

Level 1-no drusen/hard drusen only (not enrolled in the study if both eyes were at this level);

Level 2-intermediate drusen AND/OR

hyperpigmentation without hypopigmentation;

Level 3-soft drusen (distinct or indistinct) OR

hypopigmentation (with or without hyperpigmentation);

Level 4-soft drusen (distinct or indistinct) AND

pigmentary abnormalities (hyper- or hypopigmentation);

Level 5-geographic atrophy;

Level 6-neovascular AMD.

Based on previous findings, we assumed more macular disease and a higher risk of developing late AMD with each successive level. ${ }^{18-20}$

\section{Identification of cases eligible for the progression study}

Participants were eligible to enrol in this progression study if their baseline photodocumentation was assessed 
as having early AMD lesions including intermediate, or soft drusen (distinct or indistinct), or retinal pigment abnormalities such as hyper- or hypopigmentation in at least one eye, as defined in the International Classification and Grading System for AMD. ${ }^{13}$

Participants who had late AMD in both eyes were not eligible to take part in this progression study. However, if only one eye had late AMD, then the fellow eye was used to assess progression.

The original baseline retinal photographs were regraded to avoid intergrader variability and time-related drift in grading over the years. Regrading of the baseline fundus photographs and the grading of the follow-up photographs was carried out by two trained graders. Intermittent sessions of regrading photographs were carried out to evaluate inter- and intragrader reproducibility. Level of agreement was determined using the kappa statistic. The intergrader kappa value for soft drusen and pigmentary abnormalities was found to be 0.70 or greater (substantial or great) and 1.00 for late AMD. Intragrader agreement was between 0.80 and 1.00 , indicating a high level of agreement.

\section{Definition of progression}

As there is no uniformly agreed upon classification of progression of AMD lesions, we looked at three different definitions for progression. The first referred to as 'interlevel' progression was based on the eye with the more severe AMD lesions. It included cases that were stratified at level 2 at baseline and had progressed to level 3, 4, 5, or 6 by their follow-up visit.

The second definition of AMD progression referred to as 'inter- and intralevel' progression included cases that were at level 2 at baseline and reached level 3,4,5, or 6 at follow-up in either eye, and in addition, it included all cases that showed increases in size and/or number of the baseline characteristics but were not great enough to constitute a change in level of AMD in either eye.

The third definition of progression was based upon an independent and masked side-by-side comparison of the baseline and follow-up photos performed for all 254 cases. This allowed progression to be classified in those cases that appeared to have worsened by comparison.

\section{Statistical analyses}

Prevalence of baseline features was not mutually exclusive but each feature was considered regardless of whether other (more severe) features were also present, since in the vast majority of cases a number of drusen types were present at the same time in the same fundus. Age was based on that calculated at the follow-up examination. Smoking was stratified into two groups: current and past smokers and those who had never smoked with the later acting as the referent group. Progression was considered as the main outcome variable, therefore univariate logistic regression was used to determine any association between potential risk factors and AMD progression. Multivariate logistic regression was used to determine the strength of association between early AMD lesions and the progression to late AMD, controlling for such factors as age, smoking, and time of follow-up. Statistical analyses were performed using the SPSS statistical software package (SPSS Inc., Chicago, USA). A 5\% level of significance (two sided) was used. The regular intake of vitamins was not significantly associated with the progression of early AMD progression and was therefore not included in any of the multivariate analyses (data not shown).

\section{Results}

Of the 266 potentially eligible participants, 254 (96\%) cases were enrolled in the progression study. Average follow-up time was 7.2 years with $53 \%$ of participants being female and $47 \%$ having a history of current or past smoking.

The most prevalent baseline early AMD lesions were soft distinct drusen found in $45 \%(n=113)$ of cases, followed by soft intermediate drusen (37\%), hyperpigmentation (27\%), soft indistinct drusen (26\%), and hypopigmentation (18\%) (Table 1).

Progression rates over 7 years for early AMD were $24 \%$ $(61 / 254)$ for definition $1,32 \%(82 / 254)$ for definition 2 , and $33 \%(83 / 254)$ for definition 3 . These correspond with annual progression rates of $3.4,4.6$, and $4.7 \%$

respectively. For all three definitions of progression, cases that did progress were older and had a more prevalent history of smoking. Table 2 shows the baseline characteristics associated with cases that progressed and those that did not progress according to the three definitions of progression used in these analyses.

Intermediate drusen and soft indistinct drusen were the lesions with the highest progression rates of 43 and $40 \%$, respectively, over the 7 years of follow-up, equivalent to a 6.1 and $5.7 \%$ progression rate per year, respectively.

Table 3 shows progression of early AMD based on progression definition 1 ('interlevel progression'). A total of $48 \%$ of cases with AMD level 2 at baseline showed signs of progression over 7 years using (definition 1) at the final examination, compared to a $17 \%$ progression rate in cases with AMD level 3 and 15\% at level 4 .

There were $15(6 \%)$ cases that progressed from early to late AMD. In all, 10 (67\%) cases developed geographic atrophy (GA) and five (33\%) cases neovascular AMD 
Table 1 Prevalence of each early AMD feature (present in either eye) for the 254 cases

\begin{tabular}{|c|c|c|c|c|c|}
\hline \multirow[t]{2}{*}{ Drusen type at baseline } & \multicolumn{5}{|c|}{ Age (years) } \\
\hline & $\begin{array}{l}50-59 \\
(\mathrm{n}=7)\end{array}$ & $\begin{array}{c}60-69 \\
(\mathrm{n}=66)\end{array}$ & $\begin{array}{c}70-79 \\
(\mathrm{n}=125)\end{array}$ & $\begin{array}{c}80+ \\
(\mathrm{n}=56)\end{array}$ & $\begin{array}{c}\text { Total } \\
(\mathrm{n}=254)\end{array}$ \\
\hline Intermediate drusen & $0(0 \%)$ & $23(24 \%)$ & $55(58 \%)$ & $17(18 \%)$ & $95(37 \%)$ \\
\hline Soft distinct drusen & $5(4 \%)$ & $31(27 \%)$ & $58(51 \%)$ & $19(17 \%)$ & $113(45 \%)$ \\
\hline Soft indistinct drusen & $1(2 \%)$ & $11(17 \%)$ & $37(57 \%)$ & $16(25 \%)$ & $65(26 \%)$ \\
\hline \multicolumn{6}{|c|}{ Pigmentary abnormalities at baseline } \\
\hline Hyperpigmentation & $2(3 \%)$ & $19(28 \%)$ & $31(46 \%)$ & $16(24 \%)$ & $68(27 \%)$ \\
\hline Hypopigmentation & $2(4 \%)$ & $13(28 \%)$ & $21(46 \%)$ & $10(22 \%)$ & $46(18 \%)$ \\
\hline
\end{tabular}

(CNV). Only soft indistinct drusen was significantly associated with the progression from early to late AMD. Having controlled for age and smoking, our study found that those subjects with soft indistinct drusen at baseline were 3.72 (95\% CI $1.20-11.54 ; P=0.02$ ) times more likely to progress to the end stages than those without this characteristic at baseline.

In terms of retinal pigmentary abnormalities, only nonsignificant trends were seen with both cases of hyperpigmentation and hypopigmentation at baseline being at a greater risk of developing late $\mathrm{AMD}(\mathrm{OR}=2.75$ times 95\% CI 0.90-8.41 for hyperpigmentation and $\mathrm{OR}=2.43,95 \%$ CI $0.72-8.15$ for hypopigmentation) than those without these characteristics at baseline.

Of the 10 cases that developed GA by follow-up, we found that those with soft indistinct drusen at baseline were 4.12 times (95\% CI 1.06-16.0; $P=0.04$ ) more likely to develop GA than those without this lesion at baseline.

We were unable to perform similar analyses for the $\mathrm{CNV}$ cases due to the small number who developed this end stage by follow-up $(n=5)$.

In terms of AMD levels, cases having reached level 4 (soft drusen and pigmentary abnormalities) at baseline were 4.19 (95\% CI 0.88-18.24; $P=0.06$ ) times more likely to progress to the end stage of the disease than lessadvanced levels where these lesions were not present at baseline (after controlling for age and smoking).

\section{Discussion}

To have an agreed upon definition of progression would be enormously valuable when studying AMD, its risk factors, and possible interventions. We examined changes in the fundus characteristics of early AMD in a cohort of 254 participants followed for an average of 7 years to gain an understanding of the natural progression of each fundus characteristic in early AMD and the likelihood of disease progression once these features were present.
Our data show that the risk of late AMD was $0.9 \%$ per annum and the risk of early AMD progression was between 3.4 and $4.7 \%$ per annum depending on the definition of progression used. Age was a significant predictor for progression as was smoking, a finding that is consistent with the results from several large studies. ${ }^{21-24}$ The finding that age and smoking contributed to an increased risk of progression validates our definitions of progression, as this would be the anticipated finding. The fact that side by side grading agreed virtually $100 \%$ (only one case discrepancy) also indicates that the scoring system of documenting progression is valid.

In all the three definitions of AMD progression, cases that progressed had a shorter follow-up time than nonprogressed cases although the difference was significant only in definitions 2 and 3. The shorter follow-up time in progressed cases may have resulted in an underestimation of progression; however, given the small difference in average follow-up years, the underestimation would probably be minor. Conversely, given that cases that did not progress were followed longer strengthens the fact that these were in fact true cases of no progression.

The presence of soft drusen, in particular soft indistinct drusen, has been reported to be a strong predictor for progression. ${ }^{3,19,25}$ Soft indistinct drusen were present in $26 \%$ of cases at baseline of which $40 \%$ progressed over 7 years (5.7\% per year). Intermediate drusen were present in $37 \%$ of cases at baseline and these cases progressed at a rate of $43 \%$ over the seven years (6.1\% per year).

The presence of intermediate drusen at baseline was significantly greater in cases that progressed (according to all the three definitions of progression) compared to those that showed no signs of progression. Of interest, the presence of soft distinct drusen at baseline appeared to confer some degree of protection from progression to a more severe level of AMD, a significant finding that was observed for all three definitions of AMD progression 
Table 2 Univariate logistic regression analyses for the three definitions of progression

\begin{tabular}{|c|c|c|c|c|c|c|}
\hline \multicolumn{7}{|c|}{ Progression of early AMD: definition 1 (interlevel) } \\
\hline \multirow[t]{2}{*}{ Characteristics } & \multicolumn{2}{|c|}{ Nonprogressed } & \multicolumn{2}{|c|}{ Progressed } & \multirow{2}{*}{$\begin{array}{c}\text { Odds } \\
\text { ratio }(O R)\end{array}$} & \multirow{2}{*}{$\begin{array}{c}95 \% \text { confidence } \\
\text { interval }(C I)\end{array}$} \\
\hline & $\begin{array}{c}\text { Number or Mean } \\
193 / 254\end{array}$ & $\begin{array}{c}\% \text { or } S D^{\mathrm{a}} \\
76\end{array}$ & $\begin{array}{c}\text { Number or Mean } \\
61 / 254\end{array}$ & $\begin{array}{c}\% \text { or } S D \\
24\end{array}$ & & \\
\hline Age: years & 73.2 & 7.4 & 76.7 & 6. 5 & 1.07 & $1.03,1.12$ \\
\hline Smoking: ever vs never & $81 / 192$ & 42.2 & $36 / 60$ & 60.0 & 2.06 & $1.14,3.71$ \\
\hline Follow-up time: years & 7.3 & 1.1 & 7.0 & 1.0 & 0.77 & $0.58,1.02$ \\
\hline Female & $103 / 193$ & 53.4 & $32 / 61$ & 52.5 & 0.96 & $0.54,1.72$ \\
\hline Intermediate drusen ${ }^{b}$ & $58 / 193$ & 30 & $38 / 61$ & 62 & 3.85 & $2.11,7.02$ \\
\hline Soft distinct drusen ${ }^{\mathrm{b}}$ & $101 / 193$ & 52 & $12 / 61$ & 20 & 0.22 & $0.11,0.45$ \\
\hline Soft indistinct drusen ${ }^{b}$ & $50 / 193$ & 26 & $17 / 61$ & 28 & 1.11 & $0.58,2.11$ \\
\hline Hyperpigmentation $^{\mathrm{b}}$ & $57 / 193$ & 30 & $11 / 61$ & 18 & 0.53 & $0.26,1.08$ \\
\hline Hypopigmentation $^{\mathrm{b}}$ & $39 / 193$ & 20 & $7 / 61$ & 12 & 0.51 & $0.22,1.21$ \\
\hline \multicolumn{7}{|c|}{ Progression of early AMD: definition 2 (inter-and intralevel) } \\
\hline \multirow[t]{2}{*}{ Characteristics } & \multicolumn{2}{|c|}{ Nonprogressed } & \multicolumn{2}{|c|}{ Progressed } & \multirow{2}{*}{$\begin{array}{c}\text { Odds } \\
\text { ratio }(O R)\end{array}$} & \multirow{2}{*}{$\begin{array}{c}95 \% \text { confidence } \\
\text { interval }(C I)\end{array}$} \\
\hline & $\begin{array}{c}\text { Number or mean } \\
172 / 254\end{array}$ & $\begin{array}{c}\text { \% or } S D^{\mathrm{a}} \\
67.7\end{array}$ & $\begin{array}{c}\text { Number or mean } \\
82 / 254\end{array}$ & $\begin{array}{c}\% \text { or } S D \\
32.3\end{array}$ & & \\
\hline Age: years & 73.1 & 7.2 & 76.0 & 7.4 & 1.06 & $1.02,1.10$ \\
\hline Smoking: ever vs never & $69 / 171$ & 40.3 & $48 / 81$ & 59.3 & 2.15 & $1.25,3.68$ \\
\hline Follow-up time: years & 7.3 & 1.1 & 7.0 & 1.0 & 0.74 & $0.57,0.95$ \\
\hline Female & $91 / 172$ & 52.9 & $44 / 82$ & 53.6 & 1.08 & $0.64,1.83$ \\
\hline Intermediate drusen $^{\mathrm{b}}$ & $55 / 172$ & 32 & $41 / 82$ & 50 & 2.13 & $1.24,3.65$ \\
\hline Soft distinct drusen ${ }^{\mathrm{b}}$ & $89 / 172$ & 52 & $24 / 82$ & 29 & 0.39 & $0.22,0.68$ \\
\hline Soft indistinct drusen ${ }^{b}$ & $42 / 172$ & 24 & $25 / 82$ & 31 & 1.36 & $0.76,2.44$ \\
\hline Hyperpigmentation $^{\mathrm{b}}$ & $52 / 172$ & 30 & $16 / 82$ & 20 & 0.56 & $0.30,1.06$ \\
\hline Hypopigmentation $^{\mathrm{b}}$ & $34 / 72$ & 20 & $12 / 82$ & 15 & 0.70 & $0.34,1.43$ \\
\hline \multicolumn{7}{|c|}{ Progression of early AMD: definition 3 (side - by side grading) } \\
\hline \multirow[t]{2}{*}{ Characteristics } & \multicolumn{2}{|c|}{ Nonprogressed } & \multicolumn{2}{|c|}{ Progressed } & \multirow{2}{*}{$\begin{array}{c}\text { Odds } \\
\text { ratio (OR) }\end{array}$} & \multirow{2}{*}{$\begin{array}{l}95 \% \text { confidence } \\
\text { interval }(C I)\end{array}$} \\
\hline & $\begin{array}{c}\text { Number or mean } \\
171 / 254\end{array}$ & $\begin{array}{c}\% \text { or } S D^{\mathrm{a}} \\
67.3\end{array}$ & $\begin{array}{c}\text { Number or mean } \\
83 / 254\end{array}$ & $\begin{array}{c}\% \text { or } S D \\
32.7\end{array}$ & & \\
\hline Age: years & 73.1 & 7.2 & 76.0 & 7.3 & 1.06 & $1.02,1.10$ \\
\hline Smoking: ever vs never & $69 / 170$ & 40.6 & $48 / 82$ & 58.5 & 2.07 & $1.21,3.53$ \\
\hline Follow-up time: years & 7.3 & 1.1 & 7.0 & 1.0 & 0.78 & $0.61,1.00$ \\
\hline Female & $92 / 171$ & 53.8 & $43 / 83$ & 51.8 & 0.92 & $0.56,1.56$ \\
\hline Intermediate drusen $^{\mathrm{b}}$ & $54 / 171$ & 32 & $42 / 83$ & 51 & 2.22 & $1.30,3.80$ \\
\hline Soft distinct drusen ${ }^{\mathrm{b}}$ & $89 / 171$ & 52 & $24 / 83$ & 29 & 0.38 & $0.21,0.66$ \\
\hline Soft indistinct drusen ${ }^{b}$ & $41 / 71$ & 24 & $26 / 83$ & 31 & 1.45 & $0.81,2.59$ \\
\hline Hyperpigmentation $^{\mathrm{b}}$ & $51 / 171$ & 30 & $17 / 83$ & 21 & 0.61 & $0.32,1.13$ \\
\hline Hypopigmentation $^{\mathrm{b}}$ & $34 / 171$ & 20 & $12 / 83$ & 15 & 0.68 & $0.33,1.40$ \\
\hline
\end{tabular}

aSD = standard deviation.

${ }^{b}$ Early AMD features present at baseline regardless of any other feature that may also be present.

used in this study. While such findings require further investigation to understand the significance of certain drusen characteristics in AMD progression, it may be that intermediate drusen are often harbingers of soft indistinct drusen which carry a greater risk of progressing to late AMD compared to distinct drusen which may represent drusen that are associated with less risk for progression.
After controlling for age and smoking, cases with soft indistinct drusen at baseline were about four times more likely to progress to the late stages of the disease (predominantly geographic atrophy) than cases with no indistinct drusen at baseline. Having controlled for age and smoking, intermediate drusen at baseline were not significantly associated with progression from early to late AMD. The presence of hyper- or hypopigmentation 
Table 3 Proportion of cases showing progression of AMD based on definition 1 (interlevel progression) according to the AMD level at baseline

\begin{tabular}{|c|c|c|c|c|c|}
\hline \multirow[t]{2}{*}{ AMD level at baseline (column) } & \multicolumn{5}{|c|}{ AMD Level at follow-up } \\
\hline & $A M D$ level 2 & AMD level 3 & AMD level 4 & $A M D$ level 5 & AMD level 6 \\
\hline AMD level $2(n=66)$ & $34(52 \%)$ & $28(42 \%)$ & $2(3 \%)$ & $2(3 \%)$ & $0(0 \%)$ \\
\hline $\begin{array}{l}\text { AMD level } 3 \\
(n=168)\end{array}$ & $0(0 \%)$ & $142(84 \%)$ & $16(10 \%)$ & $6(3 \%)$ & $4(2 \%)$ \\
\hline $\begin{array}{l}\text { AMD level } 4 \\
(n=20)\end{array}$ & $0(0 \%)$ & $0(0 \%)$ & $17(85 \%)$ & $2(10 \%)$ & $1(5 \%)$ \\
\hline
\end{tabular}

Bold values represent cases of interlevel progression.

showed a greater than two-fold increased risk of developing late AMD although the findings were not significant.

Comparison of progression rates with other large population-based studies is difficult due to differences in the definition of the various early AMD levels used to determine progression. In particular, the inclusion/ exclusion of intermediate drusen, the incorporation of number and area of occupancy of the early AMD lesions and the incorporation of a visual acuity criterion make the results different and incomparable.

While we believe that our 'level'-based criteria used to assess progression is easily adopted and a logical way to assess AMD progression, we reanalysed our cases on the basis of the other major studies using the different AMD levels described by both the Rotterdam ${ }^{2}$ and the Agerelated Eye Disease Study (AREDS). ${ }^{6}$ The former study used the International Classification for grading AMD, while the later included intermediate drusen as an early AMD feature and incorporated a visual acuity component.

In the Rotterdam Study, where progression was defined as a worsening to the next or higher stage of AMD, we had a progression rate of $7.5 \%$ per annum (analysis not shown). The progression rate was higher than our result regardless of which definition was used. The major difference in the Rotterdam classification was the absence of a category for intermediate drusen of size 63-125 $\mu \mathrm{m}$. Our classification has been expanded to accommodate all possible lesions associated with AMD, in particular the separation of the three soft drusen types. Also, we divided pigmentary abnormalities into hyper- and hypopigmentation to allow us to assess which of the two lesions carries the greater risk of progressing. The Rotterdam study placed all pigmentary changes together. Stronger contrast between hyperpigmentation and the underlying fundus allows it to be detected much earlier than hypopigmentation, thus we placed hyperpigmentation in an earlier level than hypopigmentation.
Similarly, we applied the definitions and criteria outlined in AREDS to our data. In this case, we found an annual progression rate of $3.4 \%$ that corresponded to our 'interlevel' progression rate (analysis not shown).

Overall, each AREDS level covered an enormous range of features as well as involving a visual acuity component. For example, category 3 included characteristics anything from one large druse $(>125)$ to geographic atrophy not involving the centre of the macula. Also, some of their terminology such as 'extensive drusen' was not defined allowing for exact interpretation.

This comparison showed that variations in the definitions of progression will influence rates of progression, but only with a few percentage points.

Progression rates for AMD will be influenced by many risk factors and variables. Until we know what other environmental risk factors to measure, the best we can do is to look at the individual's fundus characteristics and predict risk based upon appearance. We have described a method for describing early AMD progression that reflects gradual progression along a 'best-informed' continuum according to current understanding of AMD and each early AMD characteristic. Soft intermediate drusen imparts the highest rate of progression and soft indistinct drusen, hyperpigmentation, and hypopigmentation each convey a different amount of risk for developing late AMD.

This paper, we hope, will lead to a better understanding of how fundus characteristics change over time, what each feature means for individual risk, and has highlighted the need for a consensus on a definition of progression if we are to be able to make the most of various interventions claiming to slow progression of AMD.

\section{Acknowledgements}

The CHARM Study is a recipient of a Project Grant from the National Health and Medical Research Council of Australia. Additional support is provided by the Perpetual Trustees Australia Ltd (Ramaciotti 
Foundation), ANZ Executors \& Trustee Company Ltd (The Hugh DT Williamson Foundation under the Medical Research and Technology in Victoria Program), The Royal Victorian Institute for the Blind, The Royal Victorian Eye \& Ear Hospital Research Committee, The Lions Club of Victoria, and The Australian Institute of Health and Welfare.

\section{References}

1 VanNewkirk MR, Nanjan MR, Wang JJ, Mitchell P, Taylor HR, McCarty CA. The prevalence of age-related maculopathy: the visual impairment project. Ophthalmology 2000; 107: 1593-1600.

2 Klaver C, Assink J, van Leeuwen R, Wolfs RC, Vingerling $\mathrm{JR}$, Stijnen T et al. Incidence and progression rates of age-related maculopathy: The Rotterdam Study. Invest Ophthalmol Vis Sci 2001; 42: 2237-2241.

3 Bressler N, Munoz B, Maguire M, Vitale SE, Schein OD, Taylor HR et al. Five-year incidence and disappearance of drusen and retinal pigment epithelial abnormalities. Waterman Study. Arch Ophthalmol 1995; 113: 301-308.

4 Klein R, Klein BEK, Jensen S, Meuer S. The five-year incidence and progression of age-related maculopathy: The Beaver Dam Eye Study. Ophthalmology 1997; 104: 7-21.

5 Sparrow J, Dickinson A, Duke A, Thompson J, Gibson J, Rosenthal A. Seven year follow-up of age-related maculopathy in an elderly British population. Eye 1997; 11: 315-324.

6 Age-related Eye Disease Study Research Group. A randomized, placebo-controlled, clinical trial of high-dose supplementation with vitamins $\mathrm{C}$ and $\mathrm{E}$, beta carotene, and zinc for age-related macular degeneration and vision loss. Arch Ophthalmol 2001; 119: 1417-1436.

7 van Leeuwen R, Klaver C, Vingerling J, Hofman A, de Jong PTVM. The risk and natural course of age-related maculopathy. Follow-up at $61 / 2$ years in the Rotterdam Study. Arch Ophthalmol 2003; 121: 519-526.

8 Robman L, McNeil JJ, Dimitrov P, Dowrick A, Tikellis G, Nicolas $\mathrm{C}$ et al. Methodology of the cardiovascular health and age-related maculopathy (CHARM) Study. Ophthal Epidemiol 2004; 11: 161-179.

9 Livingston P, Carson C, Stanislavsky Y, Lee S, Guest C, Taylor H. Methods for a population-based study of eye disease: the Melbourne Visual Impairment Project. Ophthalmic Epidemiol 1994; 1: 139-148.

10 Garrett SKM, McNeil JJ, Silagy C. Methodology of the VECAT Study: vitamin E intervention in cataract and age-related maculopathy. Ophthal Epidemiol 1999; 6: 195-208.
11 Taylor HR, Tikellis G, Robman LD, McCarty CA, McNeil JJ. Vitamin E supplementation and macular degeneration: randomised controlled trial. BMJ 2002; 325: 7354-7360.

12 Mc Neil JJ, Robman LD, Tikellis G, Sinclair M, McCarty CA, Taylor HR. Vitamin E supplementation and cataract: randomized controlled trial. Ophthalmology 2004; 111: 75-84.

13 Bird A, Bressler N, Bressler S, Chisholm IH, Coscas G, Davis $\mathrm{MD}$ et al. An international classification and GRADING system for age-related maculopathy and age-related macular degeneration. The International ARM Epidemiological Study Group. Surv Ophthalmol 1995; 39: 333-339.

14 Tikellis G, Robman LD, Harper CA, Garrett SKM, McNeil JJ, Taylor HR et al. The VECAT Study: methodology and statistical power for measurement of age-related macular features. Ophthal Epidemiol 1999; 6: 181-194.

15 Robman LD, Tikellis G, Garrett SK, Harper CA, McNeil JJ, Taylor HR et al. Baseline ophthalmic findings in the vitamin E, cataract and age-related maculopathy (VECAT) study. Aust NZ J Ophthalmol 1999; 27: 410-416.

16 Klein R, Davis M, Magli Y, Segal P, Klein BEK, Hubbard L. The Wisconsin age-related maculopathy grading system. Ophthalmology 1991; 98: 1128-1134.

17 Mitchell P, Smith W, Attebo K, Wang J. Prevalence of age-related maculopathy in Australia. The Blue Mountains Eye Study. Ophthalmology 1995; 102: 1450-1460.

18 Bressler SB, Maguire M, Bressler NM, Fine S. Relationship of drusen and abnormalities of the retinal pigment epithelium to the prognosis of neovascular macular degeneration. The Macular Photocoagulation Study Group. Arch Ophthalmol 1990; 108: 1442-1447.

19 Holz F, Wolfensberger T, Piguet B, Gross-Jendroska M, Wells JA, Minassian DC et al. Bilateral macular drusen in agerelated macular degeneration. Prognosis and risk factors. Ophthalmology 1994; 101: 1522-1528.

20 Klein R, Klein BEK, Tomany S, Meuer SM, Huang GH. Ten-year incidence and progression of age-related maculopathy. The Beaver Dam Eye Study. Ophthalmology 2002; 109: 1767-1779.

21 Klein R, Klein BEK, Linton K, DeMets D. The Beaver Dam Eye Study: the relation of age-related maculopathy to smoking. Am J Epidemiol 1993; 137: 190-200.

22 Vingerling J, Hofman A, Grobbee D, de Jong P. Age-related macular degeneration and smoking. The Rotterdam study. Arch Ophthalmol 1996; 114: 1193-1196.

23 Smith W, Mitchell P, Leeder S. Smoking and age-related maculopathy. The Blue Mountains Eye Study. Arch Ophthalmol 1996; 114: 1518-1523.

24 Chan D. Cigarette smoking and age-related macular degeneration. Optom Vis Sci 1998; 75: 476-484.

25 Azad R, Tewari H, Khosla P. Fellow eye in senile disciform degeneration of the macula. Indian J Ophthalmol 1983; 31: 878-881. 